\title{
Clinique psychiatrique et ethique du sujet*1
}

\section{Psychiatric clinic and ethics of the subject}

\author{
Mario Eduardo Costa Pereira*2 \\ Denise Maurano*3 \\ Sonia Leite*4
}

Ce travail a pour objectif celui de relativiser les critiques psychanalytiques adressées au champ psychiatrique fondées sur la conception de l'existence unitaire, absolue et sans clivage, de quelque chose qui fonctionnerait comme étant «La Psychiatrie». En montrant les tensions existant au sein même de la psychopathologie psychiatrique par l'émergence du sujet et de sa singularité, nous cherchons à indiquer des pistes pour la construction d'une psychiatrie fondée sur une éthique du sujet et de son désir.

Mots clés: Psychiatrie, éthique du sujet, psychopathologie

*1 Trabalho apresentado no dia 12/11/2016 no 4 e Colloque InterPsy, organizado pela Alfapsy - Alternative Fédérative des Associations de Psychiatrie, de 10 a 12 de novembro de 2016, no Rio de Janeiro.

*2 Universidade Estadual de Campinas - Unicamp (Campinas, SP, Br).

${ }^{* 3}$ Universidade Federal do Estado do Rio de Janeiro - UNIRIO (Rio de Janeiro, RJ, Br).

${ }^{* 4}$ Universidade do Estado de Rio de Janeiro - UERJ (Rio de Janeiro, RJ, Br). 
Les études psychanalytiques contemporaines sur la médecine en général et la psychiatrie en particulier ont tendance à adopter une perspective critique se fondant sur l'idée de la forclusion radicale du sujet du champ médico-psychiatrique. Dans cette perspective, le patient de la biomédecine contemporaine serait réduit à la condition d'objet sur lequel le médecin, en position de dépositaire socialement autorisé du savoir scientifique, appliquerait son expertise technique de diagnostic et de traitement. Dès lors, le discours du patient n'aurait d'autre intérêt que celui de servir de source d'informations et de signes d'un mal dont le fondement biologique l'exclurait de toute implication subjective dans sa propre souffrance.

Cette position critique très répandue propose alors que l'ordre médical, terme consacré par l'influent ouvrage du même titre de Jean Clavreul (1978), serait une modalité contemporaine du discours du maître, travesti de discours universitaire, ouvrant au service du capitalisme. Dans cette opération, le savoir scientifique - actualisé dans la figure du médecin devrait être appliqué sur le patient, le réduisant à l'ignorance sur soi-même et son mal. Corrélativement, le lien entre la médecine et le discours capitaliste fait que seules la productivité - ça marche comme sur des roulettes, comme l'indiquait Lacan (1972/1978) — et la capacité à participer au marché et à la consommation, fonctionnent comme références à la «normalité» et à «l'ordre»: tout ce qui les perturbe surgit comme trouble. Traiter, dans cette perspective, se définirait fondamentalement en termes de rendre l'individu apte à répondre de façon efficiente aux exigences immédiates du capitalisme à l'intérieur du champ social.

Dans son intervention La place de la psychanalyse dans la médecine (1966a/1986), Lacan attire notre attention sur la présence hégémonique du discours scientiste dans la culture et souligne ses conséquences sur le rôle du médecin et de la clinique, qui perdent dès lors leur fonction symbolique en faveur d'innombrables experts en santé, intermédiaires dans la distribution des appareils technologiques. D'où l'importance de l'éthique de la psychanalyse dans la récupération de la singularité de la rencontre clinique, comme nous le soulignerons plus loin. 


\section{ARTIGOS}

La suite de son enseignement (p. ex. Lacan, 1966b et 1969-1970/1991) rendra plus claire le fait que le discours excluant le sujet ne peut être localisé, au sens d'une origine, directement dans le champ médical, puisqu'il constitue un fait de culture, soit un discours contemporain se multipliant à partir de différents agents. Les termes par lesquels l'exprime Lacan déjà dans sa conférence de 1966 sont bien connus et montrent bien qu'il conçoit que la médecine subit les effets de la science, plus qu'elle ne les produit: «Permettez-moi d'épingler plutôt comme faille épistémo-somatique, l'effet que va avoir le progrès de la science sur la relation de la médecine avec le corps. Là encore la situation est pour la médecine subvertie du dehors»».

Lorsque les critiques psychanalytiques au champ médico-psychiatrique s'adressent à «La» psychiatrie comme une unité, la considérant comme un «ordre» stable sur la perspective structurelle, elles finissent par méconnaitre les profonds clivages et divergences, y compris éthiques, affectant ce champ, et négligent l'histoire complexe de la psychanalyse dans ses rapports à la médecine et à la psychiatrie. Quelques schématismes simplistes sous-estiment la profonde hétérogénéité de positions théorico-cliniques vives et radicalement en conflit sur la scène médico-psychiatrique actuelle. En outre, certains débats de la philosophie de la psychiatrie contemporaine sont de première importance, politiquement, pour soutenir des positions non hégémoniques devant être reconnues, valorisées et approfondies.

Ce travail, qui se veut propositionnel, se déploie à partir de la question suivante: - Comment concevoir et formuler une pratique médico-psychiatrique éthiquement fondée sur le sujet du désir inconscient? Ce projet évidemment ambitieux assume les risques d'abandonner la position confortable de la critique stricto sensu. Pour l'heure, faute d'espace, nous nous contenterons de mettre en relief certains éléments fondamentaux de l'histoire et des débats contemporains sur la place du sujet dans la médecine, lesquels nous permettrons de tracer les grandes lignes pour constituer ce que nous appellerons une «psychiatrie fondée sur le sujet». Du point de vue éthique et politique, nos efforts cherchent à offrir des ressources théoriques, métapsychologiques et cliniques positives et concrètes à tous ceux qui, travaillant dans le champ hétérogène de la dite «santé mentale», luttent au quotidien pour soutenir dans leurs pratiques une position de respect et d'écoute de la singularité du sujet du désir inconscient.

Dans l'édition de septembre 2014 de l'important British Journal of Psychiatry, en un éditorial intitulé «Psychopathology as the basic science of psychiatry», Giovanni Stanghellini défend l'idée que la science biomédicale 
s'est en partie construite sur la transformation de la plainte en symptôme, artifice qui lui aurait permis «de voir dans la plainte (l'épuisement, par exemple) l'effet d'une cause pathologique active dans le corps humain (une dysfonction endocrinienne, par exemple).» Cependant, comme l'avait déjà fait Lacan dans sa conférence sur la place de la psychanalyse dans la médecine, il développe son argument en montrant la différence profonde séparant demande, désir et jouissance. Voyons en quels termes Stanghellini s'exprime:

Ce déplacement de la plainte vers le symptôme puis vers la pathophysiologie peut avoir éclipsé le fait que la plainte a une signification pour l'individu qui souffre: elle exprime une question ou un désir.

Et il conclut son propos par un exemple clinique absolument frappant:

Une personne peut ne pas obligatoirement chercher à éliminer sa plainte, mais plutôt à réaliser son désir (voir son médecin échouer pour triomphalement devenir son patient incurable, par exemple).

Cette reconnaissance dans l'éditorial d'un des plus importants périodiques psychiatriques contemporains de l'incidence du sujet de l'inconscient sur le champ de la clinique, allant même à concevoir comme nécessaire pour la psychopathologie la distinction entre les plans de la demande, du désir et de la jouissance, démontre bien que, telle comme «La» femme, en tant que totalité, «La» psychiatrie, non plus, n'existe pas.

En réalité, l'hétérogénéité des positions concernant les relations entre sujet et psychopathologie constitue un des éléments centraux de l'histoire de la psychiatrie. La Psychopathologie générale de Jaspers (1979), par exemple, exprimait déjà dans son «Introduction» des sérieuses réserves envers une pratique psychiatrique centrée sur le diagnostic plutôt que sur le patient lui-même. Ainsi y lit-on: «Le diagnostic vient en dernier lieu dans la compréhension psychiatrique d'un cas». Pour Jaspers, le diagnostic devrait découler d'un rigoureux processus d'investigation du vécu subjectif du patient, jamais le remplacer.

À partir de 1959, dans les fameux séminaires de Zollikon qu'il destine à un public fondamentalement constitué de psychiatres, Martin Heidegger (2001) soutenait que toutes les représentations encapsulées d'une psyché, d'un sujet objectivable, d'une personne, d'un moi utilisées en psychopathologie devraient disparaître. Cet auteur, qui a eu autant d'influence sur Lacan, considérait que la Psychologie, l'Anthropologie et la Psychopathologie objectalisaient l'homme et le réduisaient à sa dimension ontique, à la totalité de ce qui se vérifie dans le 


\section{ARTIGOS}

champ de l'expérience. Dans cette optique, la science moderne se fonderait sur la contradiction de prétendre tout objectiver, y compris le subjectif (Heidegger, 1954/2002). Ce qui devient encore plus inquiétant lorsqu'il s'agit de la transposition des savoirs scientifiques sur le plan concret de la clinique, forcément impliquant des sujets et leurs existences effectives.

Lacan n'a pas été le seul à boire à la source heideggerienne pour penser la dimension proprement humaine et singulière de la psychopathologie. Nombre de médecins et psychiatres, beaucoup ayant reçu une formation psychanalytique, l'ont également fait, certains avant lui. Que l'on pense à Ludwig Binswanger, psychiatre suisse, fortement influencé par la théorie freudienne, mais aussi par les idées de Husserl et de Heidegger, propose l'idée d'une «anthropologie psychiatrique» comme discipline fondamentale pour interroger ce que tomber mentalement malade signifie comme phénomène spécifique de l'Homme en tant que tel. La psychopathologie est donc, pour cet important psychiatre, liée à l'existence humaine prise comme être-dans-lemonde plutôt qu'à la description de soi-disant «maladies mentales».

L'Anthropologie médicale/psychiatrique constitue donc une déjà traditionnelle investigation épistémologique et éthique visant à définir le statut proprement humain de l'activité clinique. Elle ne nie pas la légitimité, pour la Médecine, de rechercher ses ressources opératoires dans les dites sciences fondamentales (biologie, physiologie, ...), mais son but est de repositionner le patient dans sa singularité au cœur de l'activité thérapeutique.

C'est cette perspective qui permettra à Binswanger, par exemple, de chercher dans la schizophrénie le sens anthropologique et subjectif d'une existence accomplie et ses formes d'empêchement et d'échec, plutôt que les symptômes d'une maladie mentale. À ses yeux, le champ spécifique de l'intervention médico-psychiatrique serait de diriger le traitement non pas vers une «normalisation» ou adaptation à la norme sociale, mais vers la restitution des conditions de possibilité d'une existence accomplie depuis la perspective subjective et singulière de chacun.

L'Anthropologie médicale surgit comme une discipline autonome au travers de l'œuvre d'un autre auteur fortement influencé par la lecture de Freud: Viktor von Weizsäecker (1886-1957). Ce grand neuro-psychophysiologue avait pour dessein de décrire la «pathologie» humaine non pas en matière de phénomènes naturels, mais au moyen de catégories existentielles et proprement «pathiques». Pour lui, il était «impératif d'incorporer la subjectivité du patient à la réalité objective devant être traitée». Ainsi, «comme notre tâche n'est pas de retirer le sujet de son propre circuit, l'objectivité de l'art médical 
doit être complètement repensée». En 1926, Von Weizsäecker a été le premier à revendiquer l'introduction de la catégorie de «sujet» pour délimiter le champ $\mathrm{du}$ «pathologique». Trente ans plus tard, dans son ouvrage le plus important - Patosophie (1956) —, il affirme: «L'homme est une chose qui a un sujet et c'est cette subjectivité qui fait obstacle à tout énoncé objectif».

En 1958, le jeune Michel Foucault, alors qu'il prépare sa thèse sur l'histoire de la folie, s'enchante à la découverte de l'œuvre de von Weizsäecker au point de traduire en français un de ses livres fondamentaux: «Le cercle de la structure» (Der Gestaltkreis). Ce n'est personne d'autre qu'Henri Ey qui en signe la préface, donnant la lecture suivante à la proposition de von Weizsäecker: «... Le sujet y apparaît comme forme et contenu même de cette structure, dont le mouvement fait disparaître l'opposition entre la "psyché" et la "physis", au cœur même de l'existence humaine qui assume une rénovation perpétuelle de ses relations subjectivo-objectives». En fait, dans cet ouvrage, von Weizsäecker indique que «Tous les efforts tentés pour définir, et peut-être surmonter, la différence entre le biologique et l'inorganique finissent par tourner autour de la subjectivité. L'objet du biologiste est précisément un objet habité par un sujet». La question qui se pose alors est donc de savoir comment la conception lacanienne du «sujet» pourrait contribuer à réélaborer le complexe et hétérogène champ médico-psychiatrique à partir d'une psychopathologie organisée autour de ce concept.

Autrement dit, nous sommes de nos jours face au défi de relire l'anthropologie médicale - et ses conséquences éthiques et cliniques - à partir de la conception lacanienne du «sujet». La proposition de von Weizsäcker a eu une influence énorme sur le champ psychiatrique et sur d'innombrables psychiatres-psychanalystes d'orientation lacanienne, comme Jacques Schotte.

Pour ce dernier, le psychopathologique implique la condition humaine, et il s'agit donc d'un fait de langage. Il cherche à élaborer une anthropologie philosophique fondée sur la psychanalyse, capable de placer l'anthropos, l'homme, au cœur de la clinique psychiatrique. Telle posture diffère radicalement de la position de la psychiatrie nord-américaine d'une clinique centrée sur un diagnostic formulé en termes pragmatiques, opérationnels, dont l'intention est de s'instrumentaliser pour abolir le symptôme, même si cela entraîne également l'abolition du sujet. Comme l'annonçait la publicité de la lobotomie visant à rétablir les traumatisés de la Seconde Guerre Mondiale: «Si votre mémoire vous rend malheureux, extirpez-la et vous serez heureux». Ce qui était fait en une procédure ambulatoire simple, pour la modeste somme 


\section{ARTIGOS}

de 25 dollars, comme nous le montre Richard Ledes dans son film $A$ hole in one (2005), qui contient des scènes d'archives de l'époque.

Comme nous l'avons vu, même dans le contexte psychiatrique actuellement prédominant, les tensions entre un réductionnisme explicatif de nature neurobiologique et une conception proprement humaine et subjective de la psychopathologie émergent en tension permanente.

Il convient de rappeler, par exemple, la création, en 2005, par la World Psychiatry Association elle-même, de la dite «psychiatrie centrée sur la personne», en opposition à la psychiatrie axée sur le diagnostic psychiatrique. Corrélativement, 1'IGDA - International Guidelines for Diagnosis Assessment - a été créé comme proposition visant à introduire les «formulations idiographiques» dans les systèmes de diagnostic psychiatrique. Ce dispositif cherche une solution pour que le dossier diagnostic puisse rendre compte de la dimension unique de chaque patient, tel qu'il se montre lors de l'entretien psychiatrique courant.

L'on prévoit également, pour bientôt, la publication de la traduction brésilienne de la Classification française de troubles mentaux de l'enfant et de l'adolescent (C.F.T.M.E.A.). Fruit du labeur d'un groupe de travail mené par le psychiatre et chercheur Roger Misès, ce système nosographique, dont la première version date de 1983, est conçu pour que les dimensions psychopathologique, psychodynamique et contextuelle soient effectivement prises en compte lors du diagnostic et ne soient pas réduisent à de simples compléments de l'axe morbide central. Notons donc qu'il ne s'agit pas d'une transformation banale de la conception catégorielle des entités diagnostiques, mais d'une perspective plus dimensionnelle. Dans la C.F.T.M.E.A., c'est la notion même de diagnostic psychiatrique qui se modifie pour ce qui est des nosographies inspirées du DSM. En psychiatrie infantile et adolescente, il est indispensable que le développement en cours fasse partie du processus diagnostic. Parallèlement, dans ces étapes de l'existence humaine, la participation effective des contextes biologique, familial et social est bien plus évidente aussi bien dans l'installation des processus psychopathologiques, que dans leur possible transformation.

Parmi tant d'autres initiatives non hégémoniques, quoiqu'importantes et significatives, d'introduction de perspectives alternatives au mainstream psychiatrique, celles-ci indiquent des ouvertures sur la question du sujet tel qu'il surgit dans des pratiques cliniques en santé mentale. En ce sens, reconnaître l'importance de l'éthique du sujet dans la clinique, c'est également reconnaître, suivant Freud dans sa Conférence Psychiatrie et psychanalyse 
(1917), qu'il n'existe pas de conflit entre ces disciplines. Ce ne sont souvent que certains psychiatres et psychanalystes qui s'opposent. Plus radicalement, Freud conçoit que la psychanalyse doit fournir les fondements métapsychologiques pour la psychopathologie à l'usage des psychiatres.

Il convient de rappeler que la notion même de sujet, dont la psychanalyse devient l'héritière, surgit dans l'histoire de la pensée à partir de la crise de Descartes qui, face à l'impasse de «distinguer le vrai d'avec le faux», est, comme il le dit lui-même dans son «Discours de la méthode», «contraint d'en parler» et, voulant «marcher avec assurance en cette vie», se juge lui-même, mettant en évidence une division subjective, encore que dans le but de la dépasser et d'éliminer l'erreur à laquelle la subjectivité pourrait l'induire. Cependant, apeuré par la netteté et la clarté que peuvent présenter nos imaginations et nos rêves, c'est Dieu, l'Être parfait, que l'ampleur de cette entreprise lui fit rechercher comme garant de la vérité.

Nous pourrions donc peut-être dire que c'est la crise de Descartes qui a poussé Freud, dans sa quête de ce qui l'a inaugurée, soit le doute, l'hésitation, à mener une recherche l'ayant conduit à une autre approche de la vérité: la vérité du désir que dévoile un sujet balançant toujours entre le vrai et le faux, ou plutôt toujours assis sur des vérités qui le dépassent et qu'il ne peut saisir entièrement.

Cette approche de la vérité nous confronte à l'existence d'un réel inexorable, lequel ne saurait être saisi par quoi que ce soit. Ce qui a des implications éthiques sur toute intervention étant, ne serait-ce que peu, affectée par la perspective freudienne.

Ainsi défendons-nous non pas une application de la psychanalyse, ou de la notion de sujet lacanien à la psychiatrie, mais la possibilité d'y faire apparaître la visée de l'éthique de la psychanalyse, aussi bien dans le plan clinique, que dans les constructions théoriques, en tant que ce qui éveille à la vérité habitant le rêve, également corrélative de la vérité de la folie. Et cela vaut pour les interférences que nous faisons dans le cadre public aussi bien que privé.

\section{References}

Binswanger, L. (1977). Três formas de existência malograda - extravagancia, excentricidade, amaneiramento. Rio de Janeiro, RJ: Zahar. (Trabalho original publicado em 1956).

Clavreul, J. (1978). L'ordre médical. Paris: Seuil.

Descartes, R. (2000), Discurso do método. Rio de Janeiro: Nova Cultural (Os pensadores. (Trabalho original publicado em 1637). 


\section{ARTIGOS}

Ey, H. (1958). Préface. In Von Weizsäcker, V. Le cycle de la structure (trad. M. Foucault). Paris: Desclée de Brouwer.

Feys, J.-L. (2009). L'anthropopsychiatrie de Jacques Schotte. Une introduction. Paris: Hermann.

Freud, S. (1977). Conferência XVI. Psicanálise e psiquiatria. In Edição Standard Brasileira das Obras Psicológicas Completas de Sigmund Freud (Vol. XVI, pp. 289-303). Rio de Janeiro, RJ: Imago. (Trabalho original publicado em 1917).

Heidegger, M. (2001). Seminários de Zollikon. Petrópolis, RJ: Vozes.

Heidegger, M. (2002). A questão da técnica. In Ensaios e conferências [Vorträge und Aufsätze]. Petrópolis, RJ: Vozes. (Trabalho original publicado em 1954).

Jaspers, K. (1979). Psicopatologia Geral. Rio de Janeiro, RJ: Atheneu.

Lacan, J. (1966b). La science et la vérité. In Ecrits (pp. 855-887). Paris: Seuil.

Lacan, J. (1974). A terceira, $7^{\circ}$ Congresso da École Freudienne de Paris, 31 de outubro de 1974, Roma. Recuperado de: <http://www.freud-lacan.com/articles/article. php?url_article=jlacan031105_2>.

Lacan, J. (1978). Du discours psychanalytique. In Lacan in Italia. Milão: La Salamandra. (Trabalho original publicado em 1972).

Lacan, J. (1986). Psicoanálisis y medicina. In Intervenciones y textos 1. Buenos Aires: Manantial. (Trabalho original publicado em 1966a).

Lacan, J. (1988). O seminário. Livro 7. A ética da psicanálise. Rio de Janeiro, RJ: Jorge Zahar. (Trabalho original publicado em 1959-1960).

Lacan, J. (1991). Le séminaire. Livre XVII. L'envers de la psychanalyse (texte établi par J.-A. Miller), Paris: Seuil, 1991. (Trabalho original publicado em 1969-1970).

Mezzich, J. et al. (2003). Essentials of the World Psychiatric Associations's International Guidelines for Diagnostic Assessment (IGDA), The British Journal of Psychiatry, supplem. May-2003.

Mises, R. et al. (2012). Classification Française des Troubles Mentaux de l'Enfant et de l'Adolescent $R$. Paris: EHESP.

Stanghellini, G., Broome, M. (2014). Psychopathology as the basic science of psychiatry (Editorial). The British Journal of Psychiatry, 205, 169-170. doi: 10.1192/bjp. bp. 113.138974

Von Weizsaecker, V. (1958). Le cycle de la structure (trad. M. Foucault). Paris: Desclée de Brouwer.

Von Weizsaecker, V. (2011). Pathosophie. Grenoble: Jérôme Millon, 2011. (Trabalho original publicado em 1956).

\section{Resumos}

(Clínica psiquiátrica e ética do sujeito)

O objetivo deste trabalho é relativizar as críticas psicanaliticas dirigidas ao campo psiquiátrico fundadas na concepção da existência unitária, absoluta e sem clivagens de algo que funcionaria como "A Psiquiatria". Ao mostrar as tensões 
existentes na psicopatologia psiquiátrica pela emergência do sujeito e de sua singularidade, buscamos indicar maneiras de construir uma psiquiatria baseada na ética do sujeito e de seu desejo.

Palavras chave: Psiquiatria, ética do sujeito, psicopatologia

(Psychiatric clinic and ethics of subject)

This work aims to relativize the psychoanalytic criticisms addressed to the psychiatric field based on the conception of the unitary, absolute and undivided existence of something that would function as "the Psychiatry". By showing the tensions existing within psychiatric psychopathology by the emergence of the subject and his/her singularity, we seek to indicate ways of constructing a Psychiatry based on an ethics of the subject and of his/her desire.

Keywords: Psychiatry, ethics of the subject, psychopathology

(Clínica psiquiátrica y ética del sujeto)

El objetivo de este trabajo es relativizar las críticas psicoanalíticas, dirigidas al campo psiquiátrico, fundadas en la concepción de una unidad absoluta y sin la disociación de algo que funcionaría como "La Psiquiatría”. Al presentar las tensiones existentes en la psicopatología psiquiátrica, debido a la emergencia del sujeto y a su singularidad, buscamos indicar maneras de construir una psiquiatría basada en la ética del sujeto y de su deseo.

Palabras clave: Psiquiatria, ética del sujeto, psicopatologia

(Psychiatrische Klinik und Ethik des Subjekts)

Ziel dieser Arbeit ist es, die gegen die Psychiatrie gerichteten psychoanalytischen Kritiken zu relativieren, welche auf dem Konzept einer angeblich einheitlichen, absoluten und spaltungsfreien „Psychiatrie“ basieren. Indem wir die Spannungen aufzeigen, die in der psychiatrischen Psychopathologie durch die Manifestation des Subjekts und seiner Singularität bestehen, versuchen wir Wege aufzuzeigen, eine Psychiatrie auf der Grundlage der Ethik und des Wunsches des Subjekts aufzubauen.

Schlüsselwörter: Psychiatrie; Ethik des Subjekts; Psychopathologie

Citação/Citation: Pereira, M. E. C., Maurano, D., \& Leite, S. (2018, março). Clinique psychiatrique et ethique du sujet. Revista Latinoamericana de Psicopatologia Fundamental, 21(1), 81-91. http://dx.doi.org/10.1590/1415-4714.2018v21n1p81.6.

Editores do artigo/Editors: Profa. Dra. Ana Maria Rudge e Profa. Dra. Sonia Leite.

Recebido/Received: 5.1.2018/ 1.5.2018 Aceito/Accepted: 20.2.2018 / 2.20.2018 


\section{ARTIGOS}

Copyright: (C) 2009 Associação Universitária de Pesquisa em Psicopatologia Fundamental/ University Association for Research in Fundamental Psychopathology. Este é um artigo de livre acesso, que permite uso irrestrito, distribuição e reprodução em qualquer meio, desde que o autor e a fonte sejam citados / This is an open-access article, which permits unrestricted use, distribution, and reproduction in any medium, provided the original authors and sources are credited.

Financiamento/Funding: Os autores declaram não terem sido financiados ou apoiados / The authors have no support of funding to report.

Conflito de interesses/Conflict of interest: Os autores declaram que não há conflito de interesses / The authors have no conflict of interest to declare.

\section{Mario Eduardo Costa Pereira}

Psiquiatra; Psicanalista; Professor titular de Psicopatologia Clínica pelo Laboratoire de Psychopathologie Clinique et Psychanalyse da Aix-Marseille Université (França); Livre-Docente em Psicopatologia do Depto de Psiquiatria da FCM/UNICAMP (Campinas, SP, Br), onde dirige o Laboratório de Psicopatologia: Sujeito e Singularidade (LaPSuS); Diretor do Núcleo de São Paulo do Corpo Freudiano - Escola de Psicanálise.

marioecpereira@uol.com.br

\section{Denise Maurano}

Psicóloga; Psicanalista; Professora titular da Universidade Federal do Estado do Rio de Janeiro - UNIRIO (Rio de Janeiro,RJ, Br), vinculada ao CCJP/Curso de Direito e no Programa de Pós-Graduação em Memória Social; Doutorado em Filosofia pela Universidade de Paris XII e Pontifícia Universidade Católica do Rio de Janeiro - PUC/ RJ (Rio de Janeiro, RJ, Br); Pós-doutorado em Letras pela PUC/RJ; Membro do Corpo Freudiano do Rio de Janeiro e Membro Correspondente de Association Insistance (Paris). Editora de Psicanálise\&Barroco em Revista. dmauranomello@gmail.com.br

\section{Sonia LeITE}

Psicóloga; Psicanalista; Coordenadora Adjunta da Residência Multiprofissional em Saúde Mental(RMSM) SES/UERJ (Rio de Janeiro,RJ, Br); Professora e supervisora da RMSM; Doutora em Psicologia Clínica pela Pontifícia Universidade Católica do Rio de Janeiro PUC/RJ (Rio de Janeiro, RJ, Br); Editora da Revista Latinoamericana em Psicopatologia Fundamental; Membro do Corpo Freudiano Escola de Psicanálise Seção RJ; Coordenadora de Ensino do Corpo Freudiano Escola de Psicanálise - Seção Rio de Janeiro. soniacleite@uol.com.br

This is an open-access article, which permits unrestricted use, distribution,

BY-NC and reproduction in any medium for non-commercial purposes provided the original authors and sources are credited. 